\title{
Quantitative analysis of delocalization in the vicinity of the metal-insulator transition in doped semiconductors
}

\author{
I Shlimak $\dagger$, M Kaveh $\dagger, \mathrm{R}$ Ussyshkin $\dagger$, V Ginodman $\dagger$, L Resnick $\dagger$ and \\ V F Gantmakherł \\ $\dagger$ Jack and Pearl Resnick Institute of Advanced Technology, Department of Physics, Bar- \\ Ilan University, Ramat-Gan 52900, Israel \\ $\ddagger$ Institute of Solid State Physics, Russian Academy of Sciences, 142432 Chernogolovka, Russia
}

Received 30 June 1997

\begin{abstract}
A temperature-induced crossover from hopping to metallic conductivity is observed for barely insulating samples of Ge:As and Ge:Sb with impurity concentration $N$ just below the critical value $N_{c 0}$. The values of the correlation length $\xi$ are obtained on both sides of the transition. A method is developed for determination of a 'delocalization temperature' $T_{d}$, which separates the hopping and metallic conductivity. It is shown that the dependence of $T_{d}$ on $N$ for various semiconductors can be reduced to a universal curve using normalization of the $T$-scale by the mean energy of the Coulomb interaction $W=\left(e^{2} / \kappa_{0}\right) N_{c 0}^{1 / 3}$.
\end{abstract}

The metal-insulator transition (MIT) is defined by the vanishing of the dc conductivity at zero temperature [1]: the value of $\sigma(0) \equiv \sigma(T \rightarrow 0)$ when plotted as a function of the impurity concentration $N$, is equal to zero on the insulating side of the MIT and remains finite on the metallic side, obeying scaling behaviour in the vicinity of the transition [2]:

$$
\sigma(0)=\gamma \frac{e^{2}}{\hbar} \frac{1}{\xi} \propto\left(\Delta N / N_{c 0}\right)^{\mu} .
$$

Here $\gamma=1 / 3 \pi^{2}$ [3], $\xi$ is the correlation length, $N_{c 0}$ is the critical impurity concentration, $\Delta N=N-N_{c 0}$, and $\mu$ is the critical conductivity exponent which is to be determined from experiments. The scaling behaviour of $\sigma(0)$ is conditioned by the divergence of $\xi$ when the transition is approached: $\xi \propto\left(\Delta N / N_{c 0}\right)^{-\mu}$.

The zero-temperature conductivity does not behave as an ordinary metallic Drude conductivity in the immediate vicinity of the transition point. This happens only when it reaches the Mott minimal conductivity

$$
\sigma_{m}=C_{0}\left(e^{2} / \hbar\right) N_{c 0}^{1 / 3}
$$

i.e. only when $\xi$ reduces to the average inter-impurity distance $N_{c 0}^{-1 / 3}$. Here $C_{0}$ is a numerical coefficient. Mott has defined its value approaching $\sigma_{m}$ from the metallic side. When approaching $\sigma_{m}$ from below, it is natural to apply the scaling theory and assume $C_{0}=\gamma$.

The conductivity $\sigma(0)<\sigma_{m}$ represents a special kind of conductivity [2], which has no clear model representation and can be named critical, or scaling, or quantum conductivity. So, we suggest that the $\mathrm{N}$-axis can be divided into three regions: insulating (I), scaling $(\mathrm{S})$, and metallic $(\mathrm{M})$. Since in the S-region $\sigma(0) \neq 0$, the S-region must be also regarded 
as metallic and the MIT takes place on the boundary between the I- and the S-regions. With concentration $N$ in the I-region, the Fermi level is situated in the localized part of the density of states; in the S-region, it is in its delocalized part.

Non-zero, but low, temperature results for delocalized electrons of the S-region in finite phase relaxation time $\tau_{\varphi}$ and finite phase relaxation length $L=\left(D \tau_{\varphi}\right)^{1 / 2}(D$ is the diffusion coefficient). According to [1] and [4], $\sigma(T)$ in the S-region can be expressed in the form

$$
\sigma(T)=\gamma \frac{e^{2}}{\hbar \xi}+\beta \frac{e^{2}}{\hbar L(T)} .
$$

Here $\beta=2 / 3 \pi^{3}=0.022$ and $\gamma=1 / 3 \pi^{2}=0.034$ are the numerical constants [3]. (We shall assume, for simplicity, $\gamma \approx \beta$.) For localized electrons of the I-region, because of the phonons, a finite conductivity appears which is determined by the variable-range-hopping mechanism [5] and characterized by an exponential temperature dependence.

It is now widely accepted that in the immediate vicinity of the MIT, the electronelectron (e-e) interaction plays the leading role. For the S-region, this means that it is the e-e interaction that determines $\tau_{\varphi}$ and $L$ [6]:

$$
\tau_{\varphi}=\tau_{e e}=\hbar / T \quad \text { and } \quad L=(D \hbar / T)^{1 / 2} .
$$

For the I-region, e-e interaction produces the soft Coulomb gap at the Fermi level and brings the exponential dependence of $\sigma(T)$ to the form of the Shklovskii-Efros law [5]

$$
\sigma(T)=\sigma_{0} \exp \left[-\left(T_{S E} / T\right)^{1 / 2}\right] \quad T_{S E}=2.8 e^{2} / \kappa \xi .
$$

Here $\sigma_{0}$ is a parameter and the dielectric constant $\kappa$ is equal to $\kappa_{0}$ far from the MIT and diverges at the point of the MIT [7]:

$$
\kappa=\kappa_{0}+4 \pi e^{2} g_{F} \xi^{2}
$$

where $g_{F}=(\partial N / \partial \epsilon)_{\epsilon_{F}}$ is the density of states at the Fermi level.

In both regions around the MIT, I and S, the increase of the temperature leads to crossovers in conducting mechanisms.

Substituting $L$ from (4) into (3) and using the Einstein relation $\sigma(T)=e^{2} g_{F} D$, we obtain the following equation in dimensionless units $x=\sigma(T) / \sigma(0), t=T / T^{*}$, $T^{*}=\beta / \xi^{3} g_{F}[8]:$

$$
x^{3 / 2}=x^{1 / 2}+t^{1 / 2} .
$$

In the low-temperature limit $T \ll T^{*}, x \rightarrow 1$ and the solution of (7) is

$$
x=1+t^{1 / 2} \quad \sigma(T)=\sigma(0)+m T^{1 / 2} \quad m=\beta^{1 / 2}\left(e^{2} / \hbar\right) g_{F}^{1 / 2} \xi^{1 / 2} .
$$

In the opposite limit $T \gg T^{*}$, from $\sigma(T) \gg \sigma(0)$ it follows $[4,9,10]$ that

$x=\frac{2}{3}+t^{1 / 3} \quad \sigma(T)=a+b T^{1 / 3} \quad a=\frac{2}{3} \sigma(0) \quad b=\beta^{2 / 3}\left(e^{2} / \hbar\right) g_{F}^{1 / 3}$.

The equation $T=T^{*}$, i.e.

$$
T_{c r s}^{(S)}=\beta / \xi^{3} g_{F}
$$

defines the crossover line in the S-region [8]. It separates temperature regions where the relation $\Delta \sigma \propto T^{p}$ has indices $p=\frac{1}{2}$ and $\frac{1}{3}$. For $T<T_{c r s}^{(S)}$ the diffusion coefficient $D$ is determined by the static random potential and for $T>T_{c r s}^{(S)}$ by charge fluctuations.

The ' $T^{1 / 3}$-dependences' (9) have been observed in different doped semiconductors: InSb [11], GaAs [11-13], and Ge [14]. The MIT in these experiments was approached by changing both the impurity concentration $N$ ( $N$-MIT) and the magnetic field $B$ ( $B$-MIT). The measurements in Si were usually interpreted in terms of (8), as a ' $T^{1 / 2}$-dependence' 
[15-18]. It is not always simple to decide a priori what scale, $T^{1 / 2}$ or $T^{1 / 3}$, is better. We have replotted here the data of [17] and [18] on the $T^{1 / 3}$-scale. In contrast, data for Ge:Ga were presented recently in the $T^{1 / 2}$-scale [19]. However, usually both presentations give practically the same $N_{c 0}$ value.

Inserting $t=1$ into (7), one obtains $x \approx 1.75$, i.e. $\sigma\left(T^{*}\right) \approx 1.75 \sigma(0)$. This defines the self-consistent procedure for the analysis of the data in the S-region. One would expect the $T^{1 / 3}$-scale to be applicable in the nearest vicinity of the MIT only [20].

The crossover line exists also in the I-region. For variable-range hopping to occur, the hopping length $r_{S E}$

$$
r_{S E}=\frac{1}{4} \xi\left(T_{S E} / T\right)^{1 / 2}
$$

must be longer than $\xi$. From the equality $r_{S E}=\xi$ and from expressions (5) for $T_{S E}$ and (6) for $\kappa$, the equation for the second crossover line follows

$$
T_{\text {crs }}^{(I)}=\frac{\beta^{\prime} / \xi^{3} g_{F}}{1+\kappa_{0} / 4 \pi e^{2} g_{F} \xi^{2}} \quad \beta^{\prime}=0.014 .
$$

The value of $\beta^{\prime}$ follows from the numerical coefficients in (5), (6), and (11). In the immediate vicinity of the MIT (12) becomes similar to (10) for the S-region.

The change of the conductivity along the crossover line (12) in the I-region is more fundamental as compared to the crossover in the S-region (10). In the I-region, a transition takes place from the exponential to the power law in the temperature dependence of the conductivity. The conductivity has insulator-like behaviour below $T_{c r s}^{(I)}$ and metalliclike behaviour above $T_{c r s}^{(I)}$. We shall refer to this effect as to a temperature-induced metal-insulator transition ( $T$-MIT). In this language, the critical concentration $N_{c 0}$, which separates the samples with insulating and metallic behaviours of conductivity, shifts to lower concentration with increasing $T$. With relation (1) between $\Delta N$ and $\xi$, the function $T_{c r s}^{(I)}(\xi)$ defines the 'delocalization temperature' $T_{d}$ at a given concentration $N$ and (12) gives an implicit expression for the function $N_{c}(T)$ with $N_{c}(0) \equiv N_{c 0}$.

A similar quasi-phase diagram in the vicinity of the MIT was proposed earlier in [8]. However, the correlation length $\xi$ was supposed there to be controlled by the degree of disorder. The later cannot be quantitatively measured. Therefore the diagram was compared with experiment only qualitatively. In this work we will concentrate on the classical heavily doped semiconductors $\mathrm{Ge}$ and $\mathrm{Si}$ where $\xi$ is controlled by the concentration, which can be precisely measured. We report the observation of the temperature-induced crossover from hopping to metallic conductivity in barely insulating samples of Ge:As and Ge:Sb with impurity concentrations $N$ just below $N_{c 0}$. The quantitative analysis of the vicinity of the MIT is presented: we plot the function $N_{c}(T)$ for a series of samples of Ge:As and Ge:Sb. We also analyse the data for $\mathrm{Si}: \mathrm{B}$ and $\mathrm{Si}: \mathrm{P}$ obtained by other authors. We demonstrate that the dependence of the dimensionless quantity $\left|\Delta N_{c} / N_{c 0}\right| \equiv\left[N_{c}(T)-N_{c 0}\right] / N_{c 0}$ on the reduced temperature $T / W$ merges for different impurity systems into a universal curve. Here $W=\left(e^{2} / \kappa_{0}\right) N_{c 0}^{1 / 3}$ is the mean energy of the random potential caused by the Coulomb interaction between charged impurities.

Two series of uncompensated samples of Ge metallurgically doped by As and Sb with impurity concentration close to the MIT were cut from crystals grown by the Czochralski method. The effective concentrations of impurities responsible for the low-temperature conductivity were calculated directly from the resistance measurements using the method and scale proposed in [21]. The $N$-MIT in these series of samples was described earlier in [14]. It was shown there that $N_{c 0}=3.5 \times 10^{17} \mathrm{~cm}^{-3}$ for Ge:As and $1.44 \times 10^{17} \mathrm{~cm}^{-3}$ for $\mathrm{Ge}: \mathrm{Sb}$ and that $\mu=1$ for both series. Figure 1 shows the temperature dependence of 


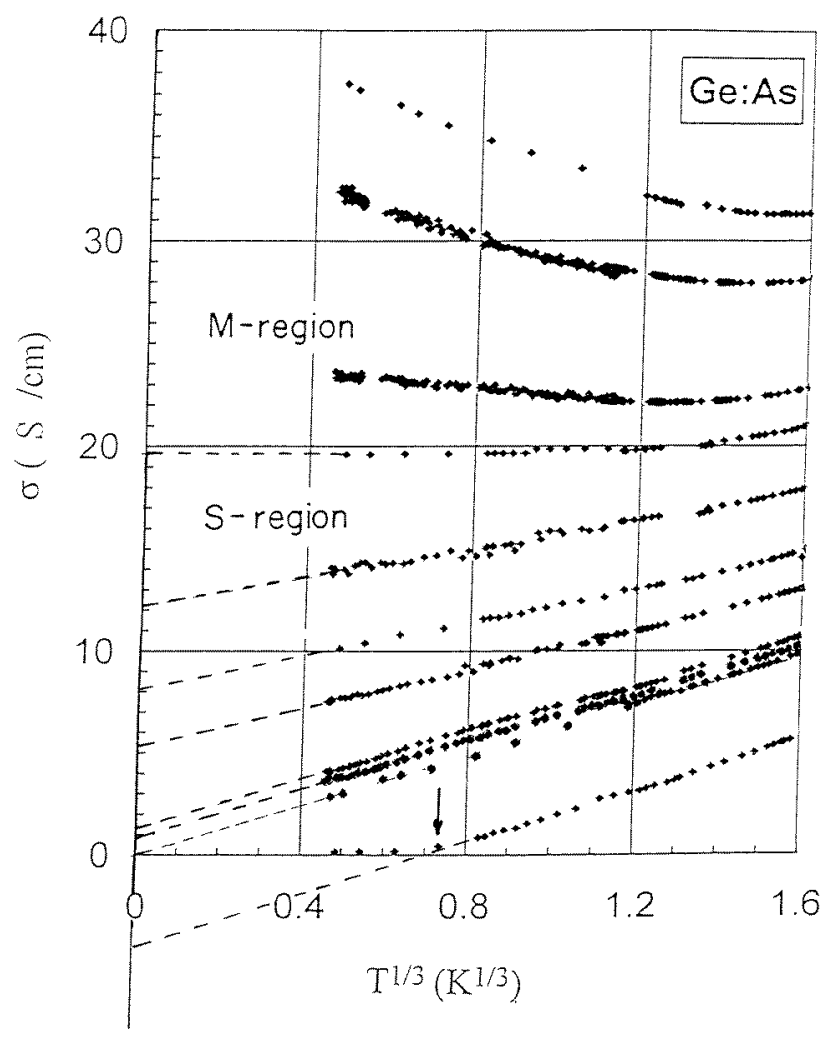

Figure 1. The temperature dependence of conductivity $\sigma(T)$ for a series of Ge:As samples. Impurity concentration from top to bottom in units of $10^{17} \mathrm{~cm}^{-3}$ : 5.38, 5.15, 4.60, 4.45, $4.17,3.91,3.82,3.58,3.56,3.50,3.00$. The dashed lines show the extrapolation procedure to $T=0$. The arrow shows the 'delocalization temperature' $T_{d}$ for the insulating sample with $N=3.00 \times 10^{17} \mathrm{~cm}^{-3}$.

conductivity $\sigma(T)$ for the series of Ge:As samples in terms of $\sigma$ against $T^{1 / 3}$. One can see that the function $\sigma(T)$ is well represented by the power law (9) not only in the metallic S-region, but in the I-region as well (see the sample with $N=3.0 \times 10^{17} \mathrm{~cm}^{-3}$ ), with the difference that in the I-region $a<0$ and the $T^{1 / 3}$-dependence is observed only at $T>T_{d}$.

Figure 2 shows the conductivity as a function of $N$ at different $T$ for these samples. We notice first the linear dependence of the limiting values of $\sigma(T \rightarrow 0)$ obtained from $\sigma(T)$ curves by extrapolation to $T=0$. It confirms that the critical index $\mu$ in (1) is equal to unity

$$
\xi \propto\left(\Delta N / N_{c 0}\right)^{-1}
$$

and gives the value of the critical concentration $N_{c 0}$. From here we receive the lower scale for the $x$-axis, in units $\Delta N / N_{c 0}$.

The linear behaviour of $\sigma(T)$ against $N$ persists at non-zero $T$ as well. We see from figure 2 that all the lines intersect in one point, at $\Delta N / N_{c 0} \approx 0.27$. At this concentration the conductivity $\sigma$ hardly depends on $T$ (see figure 1 , the sample with $N=4.45 \times 10^{17} \mathrm{~cm}^{-3}$ ). We believe that this occurs because the temperature-dependent quantum corrections to the conductivity (3) and the classical contribution of the temperature-dependent scattering, being 


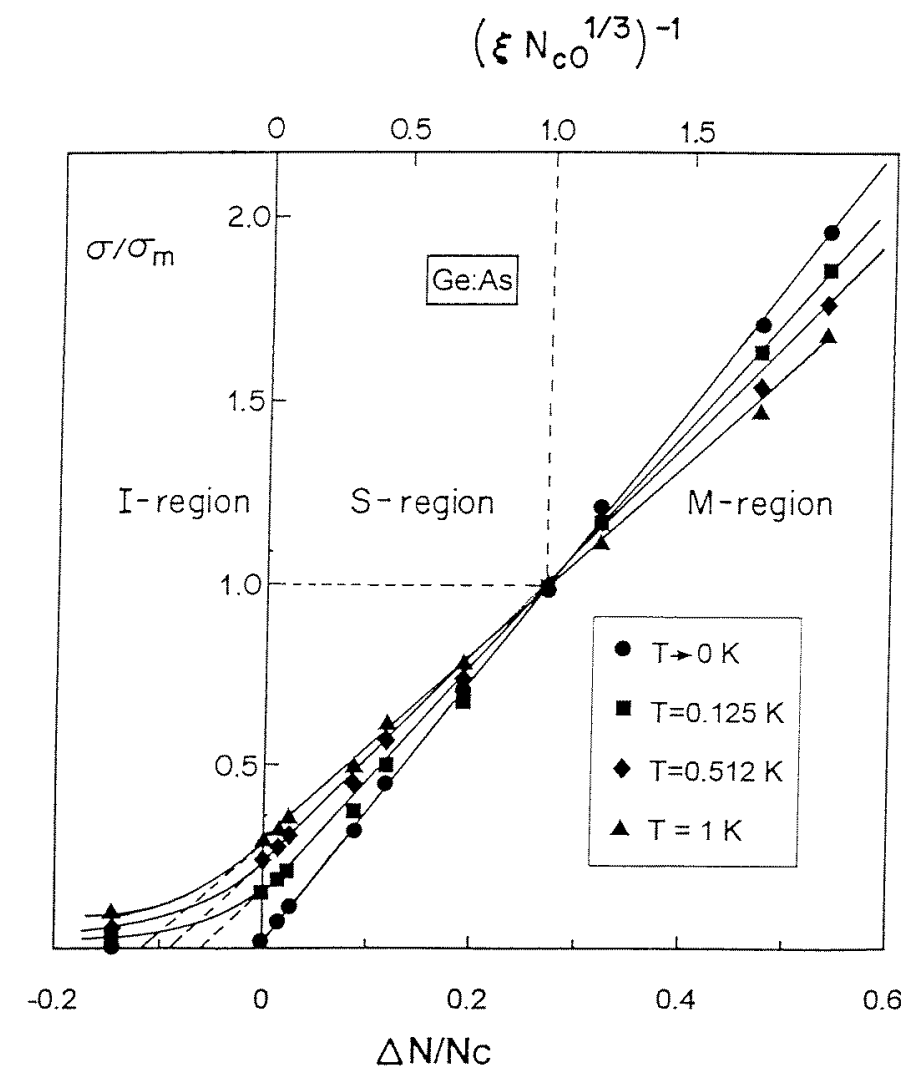

Figure 2. Dimensionless conductivity $\sigma(T) / \sigma_{m}$ as a function of dimensionless impurity concentration $\Delta N / N_{c 0}$ for a series of Ge:As samples $\left(N_{c 0}=3.5 \times 10^{17} \mathrm{~cm}^{-3}, \sigma_{m}=\right.$ $\left.C_{0}\left(e^{2} / \hbar\right) N_{c 0}^{-1 / 3}=14 \mathrm{~S} \mathrm{~cm}^{-1}, C_{0}=0.12\right)$. The top axis shows the reciprocal correlation length $\xi^{-1}$ in dimensionless units $\left(\xi N_{c 0}^{1 / 3}\right)^{-1}$.

of opposite signs, nearly compensate each other. Hence, we suggest that at this concentration we are at the border between the S- and M-regions. This gives the $1 / \xi$-scale along the $x$-axis which is plotted above: $\xi^{-1}$ is zero at $\Delta N=0$, and $\xi^{-1}=N_{c 1}^{1 / 3}$ at $\Delta N / N_{c 0} \approx 0.27$ (here $\left.N_{c 1}=1.27 N_{c 0}\right) \dagger$. The scale, according to (13), is linear with $\Delta N$. As a result, one can obtain in the S-region a coefficient $c_{s}$ between the two related scales:

$$
\left(\xi N_{c 0}^{1 / 3}\right)^{-1}=c_{s}\left(\Delta N / N_{c 0}\right) \quad c_{s} \approx 4 .
$$

(14) is valid only in the S-region: there is no cause for it to be the same in the I-region where the parameter $\xi$ is called the 'localization length' $\xi^{\text {loc }}$.

The given interpretation of the intersection point defines also the reduced scale along the $y$-axis: the conductivity at the S-M-boundary is expected to be $\sigma_{m}$; hence, the coefficient $C_{0}$ in (2) is 0.12 . This value is lower than the value of 0.3 used in [14] but it is derived straight from the experiment and is closer to the theoretical value $\gamma$ which enters the expression (1).

$\dagger$ In the case of Ge:Sb, the same effect is observed for the sample with $N=1.82 \times 10^{17} \mathrm{~cm}^{-3}$ (see figure 3 in [14]), which corresponds to the value $N_{c 1} / N_{c 0}=1.26$, very close to that for Ge:As, while for p-type Si:B [17] and Ge:Ga [19] $N_{c 1} / N_{c 0}=1.08$. It is interesting to find this ratio for other doped semiconductors. 
The straight lines $\sigma\left(\Delta N / N_{c 0}\right)$ cross the $y$-axis $N=N_{c 0}$ at $\sigma$ values

$$
\sigma=\left(e^{2} / \hbar\right) \beta^{2 / 3}\left(g_{F} T\right)^{1 / 3} .
$$

From here we can obtain the density of states $g_{F}$ just at the point of the MIT:

$$
g_{F 0}=3 \times 10^{16} \mathrm{~cm}^{-3} \mathrm{~K}^{-1} .
$$

Extrapolating the straight lines $\sigma\left(\Delta N / N_{c 0}\right)$ to $\sigma=0$, denoting the intersection point with the $x$-axis as $N_{c}(T)$ and assuming $\left(N_{c}(T)-N_{c 0}\right) / N_{c 0} \ll 0.27$ one obtains from similarity of triangles the expression

$$
\left(N_{c}(T)-N_{c 0}\right) \propto\left(g_{F} T\right)^{1 / 3}
$$

which, in view of the proportionality (13), is equivalent to the equations of the crossover lines (10) and (12). Hence, the procedure described above permits us to measure the crossover line (12) and to determine the critical concentration $N_{c}(T)$ at which, at given $T$, the leading transport mechanism changes from hopping to scaling conductivity.

The procedure of extracting the function $N_{c}(T)$ by extrapolation of the linear part of the functions $\left.\sigma(N)\right|_{T=\text { constant }}$ to $\sigma=0$ was applied to the results of our experiments with Ge:As and Ge:Sb. We applied the same procedure to the data for Si:B and Si:P obtained by other authors and published in [17] and [18]. All these results are gathered in figure 3. It is easy to see that shifts of $N_{c}$ to lower $N$ with increasing $T$ are different, being higher for materials with smaller $N_{c}$.

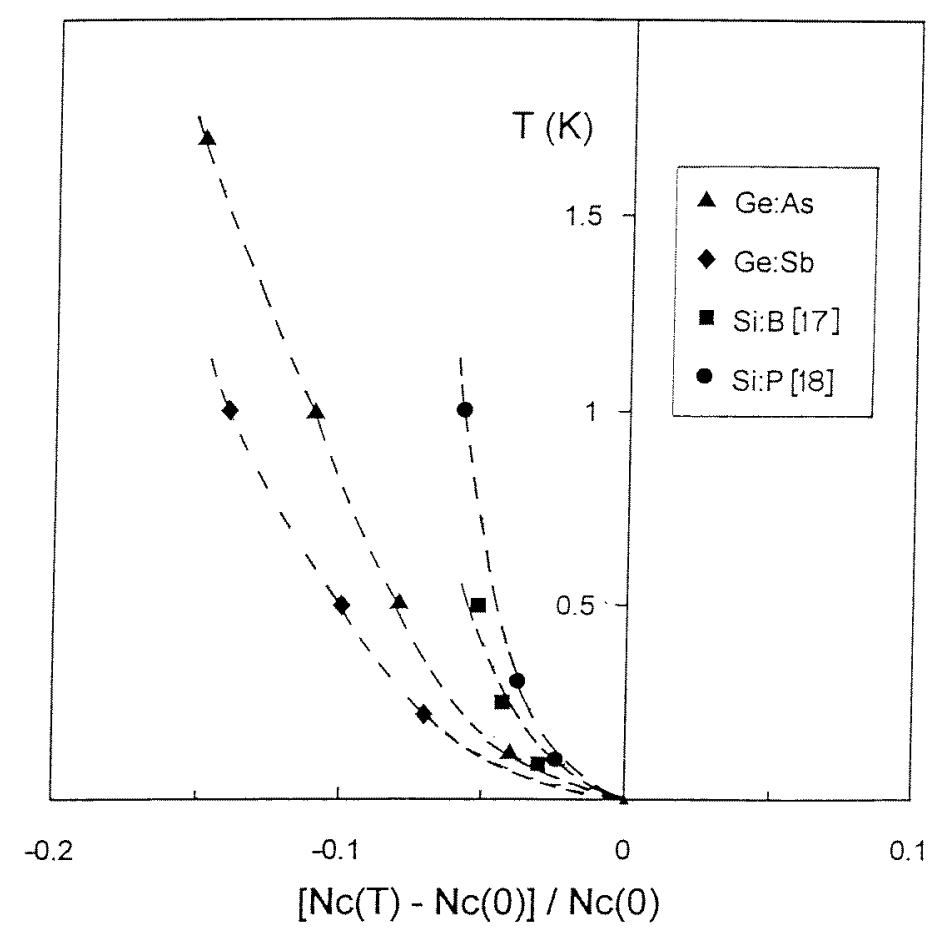

Figure 3. Dependences of $N_{c}(T)$ for Ge doped with As and Sb (present work), Si:B [17] and Si:P [18]. The dashed lines are guides to the eye. 


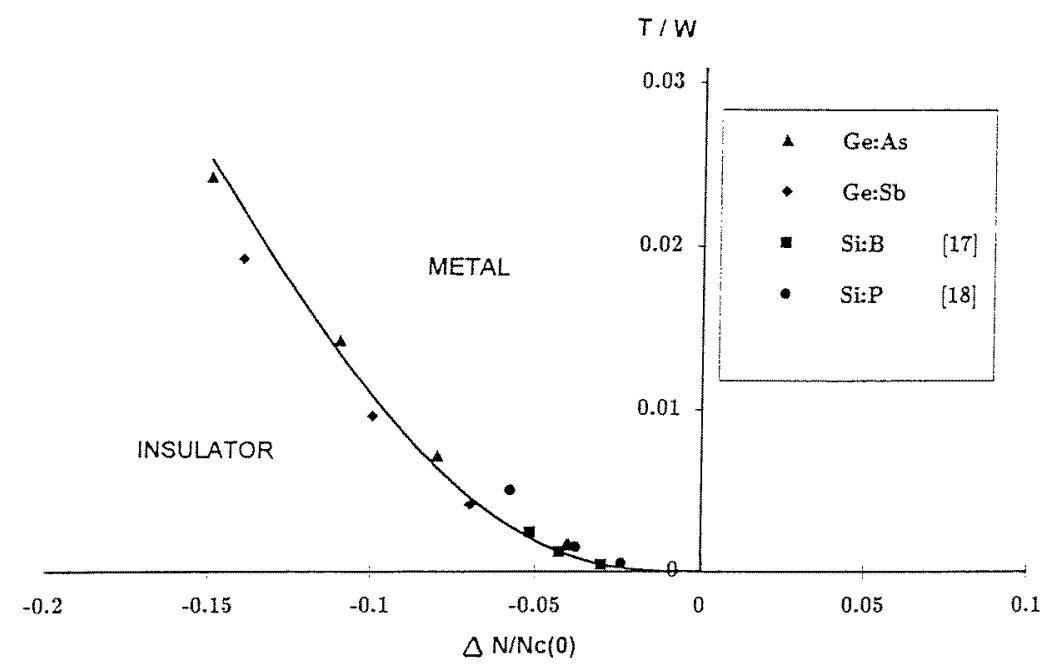

Figure 4. The vicinity of the MIT as a 'quasi-phase diagram': the normalized delocalization temperature $T / W$ as a function of the normalized impurity concentration $\Delta N / N_{c 0}$. The mean energy of the random Coulomb potential $W=\left(e^{2} / \kappa_{0}\right) N_{c 0}^{1 / 3}$ is $70 \mathrm{~K}$ for Ge:As, $52 \mathrm{~K}$ for Ge:Sb, $206 \mathrm{~K}$ for $\mathrm{Si}: \mathrm{B}$, and $200 \mathrm{~K}$ for $\mathrm{Si}: \mathrm{P}$. The solid line corresponds to the crossover temperature $T_{c r s}^{(I)}(12)$ with parameters given in the text.

To merge the data for different impurity systems into a universal curve, we reduced the temperature axis to dimensionless units, dividing $T$ by $W=\left(e^{2} / \kappa_{0}\right) N_{c 0}^{1 / 3}$, the mean energy of the random potential caused by the Coulomb interaction between charged impurities. The result is presented in figure 4: all data merge into one curve. Since $W$ is a measure of the Coulomb interaction in the impurity system, this is an argument which indicates that, indeed, this interaction governs the MIT in doped semiconductors.

Our next action is to compare the universal curve obtained with the crossover line described by (12). The solid line which fits the points is plotted in the form

$$
T=c_{1} x^{3} /\left(1+c_{2} x^{2}\right)
$$

in accordance with (12) applied to Ge:As. The value of $\kappa_{0}=16$ is used, the value of $g_{F}$ from (16) is taken as an upper limit of $g_{F}$. The numerical coefficients $\beta^{\prime}$ and $c_{i}$ between two related scales in the I-region, similar to $c_{s}$ defined in (14), are used as free parameters. The fitting gives $c_{i}=12, \beta^{\prime}=0.002$. The obtained value of $c_{i}$ is larger while the value of $\beta^{\prime}$ is smaller than corresponding values in (12) and (14). However, they are very sensitive to the value of $g_{F}$ used. We expect a further decrease of $g_{F}$ when moving inside the I-region, so that is the upper limit for $c_{i}$ and the lower limit for $\beta^{\prime}$.

To conclude, we demonstrated that the one-variable scaling theory with e-e-interactions included can be successfully applied to heavily doped semiconductors for quantitative description of the critical region of both sides of the MIT. The quantitative relations between the correlation length $\xi$ and the impurity concentration $N$ were measured. On the insulating side of the MIT, in its immediate vicinity, the conductivity has insulatorlike behaviour below some 'delocalization temperature' $T_{d}$ and metallic-like behaviour above $T_{d}$. The concentration dependence of $T_{d}$, or, in other words, the temperature dependence of the critical concentration, was measured and analysed. It turns out to be a universal curve for different doped semiconductors after the concentration is reduced 
to the critical value $N_{c 0}$ and the temperature to the mean value of the random Coulomb potential $W=\left(e^{2} / \kappa_{0}\right) N_{c 0}^{1 / 3}$.

\section{Acknowledgments}

This work was supported by the German-Israeli Foundation for Scientific Research and Development (GIF, grant No I-0319-199.07). We wish to thank Yu Galperin for discussion, $\mathrm{H}$ van Lohneysen for supplying us the data from [18] plotted in the ' $T^{1 / 3}$ ' scale, G Citver and $\mathrm{M}$ Levin for useful assistance and M Lyubalin for growing doped Ge crystals. We are very grateful to the Eric and Sheila Samson Chair of Semiconductor Technology for financial support. One of the authors (VFG) gratefully acknowledges the hospitality of the Bar-Ilan University, Israel.

\section{References}

[1] Mott N F 1990 Metal-Insulator Transitions 2nd edn (London: Taylor and Francis) Mott N F and Kaveh M 1985 Adv. Phys. 34329

[2] Abrahams E, Anderson P W, Licciardello D C and Ramakrishnan T V 1979 Phys. Rev. Lett. 42673

[3] Kawabata A 1984 J. Phys. Soc. Japan 342169

[4] Imry Y 1981 J. Appl. Phys. 521817

[5] Shklovskii B I and Efros A L 1984 Electronic Properties of Doped Semiconductors (Berlin: Springer)

[6] Altshuler B L and Aronov A G 1985 Electron-Electron Interactions in Disordered Systems ed A L Efos and M Pollak (Amsterdam: North-Holland) p 1

[7] Lee P A and Ramankrishnan T V 1985 Rev. Mod. Phys. 57287

[8] Gantmakher V F, Zverev V N, Teplinskii V M and Barkalov O I 1993 JETP 76714

[9] Imry Y and Ovadyahu Z 1982 J. Phys. C: Solid State Phys. 15 L327

[10] Altshuler B L and Aronov A G 1983 JETP Lett. 37410

[11] Newson D J and Pepper M 1986 J. Phys. C: Solid State Phys. 193983

[12] Malliepaard M C, Pepper M, Newbury R, Frost J E F, Peacock D C, Ritchie D C and Jones G A C 1989 Phys. Rev. B 391430

Malliepaard M C, Pepper M, Newbury R and Hill G 1988 Phys. Rev. Lett. 61369

[13] Friedland K-J, Ionov A N, Rentzsch R, Gladun C and Vinzelberg H 1990 J. Phys.: Condens. Matter 23759

[14] Shlimak I, Kaveh M, Ussyshkin R, Ginodman V and Resnick L 1996 Phys. Rev. Lett. 771103

[15] Thomas G A, Kawabata A, Ootuka Y, Katsumoto S, Kobajashi S and Sasaki W 1982 Phys. Rev. B 262113 Thomas G A, Paalanen M and Rosenbaum T F 1983 Phys. Rev. B 273897

[16] Shafarman W N, Koon D W and Castner T G 1989 Phys. Rev. B 401216

[17] Dai P, Zhang Y and Sarachik M P 1991 Phys. Rev. Lett. 661914

Dai P, Zhang Y and Sarachik M P 1991 Phys. Rev. Lett. 67136

Dai P, Zhang Y and Sarachik M P 1992 Phys. Rev. B 453984

[18] Stupp H, Hornung M, Lakner M, Madel O and Lohneysen H van 1993 Phys. Rev. Lett. 712634

[19] Itoh K M et al 1996 Phys. Rev. Lett. 774058

[20] Teplinskii V M, Gantmakher V F and Barkalov O I 1992 JETP 74905

[21] Shlimak I, Ussyshkin R, Resnick L and Ginodman V 1995 Appl. Phys. A 61115 